DOI: 10.1002/ ((please add manuscript number))

Article type: Essay

\title{
Graphene-based biosensors: Going simple
}

Eden Morales-Narváez, Luis Baptista-Pires, Alejandro Zamora-Gálvez and Arben Merkoçi*

Dr. Eden Morales-Narváez, Luis Baptista-Pires, Alejandro Zamora-Gálvez, Prof. Arben Merkoçi

Catalan Institute of Nanoscience and Nanotechnology (ICN2), CSIC and The Barcelona Institute of Science and Technology, Campus UAB, Bellaterra, 08193 Barcelona, Spain E-mail: arben.merkoci@icn2.cat

Prof. Arben Merkoçi

ICREA, Pg. Lluís Companys 23, 08010 Barcelona, Spain.

Keywords: graphene, biosensors, flexible substrates, market

We critically discuss the main properties of graphene derivatives facilitating optical and electrical biosensing platforms and how the integration of graphene derivatives, plastic and paper can lead to innovative devices in order to simplify biosensing technology and manufacture easy-to-use, yet powerful electrical or optical biosensors. We also underscore some crucial issues to be overcome in order to bring graphene-based biosensors to the market.

\section{Introduction}

Technologies facilitating diagnostics, drug discovery, food safety, defense, security, and environmental monitoring are of great interest to industry, government initiatives and research scientists. Biosensing has become an important approach within these technologies, particularly those biosensors integrating highly desirable characteristics such as specificity (suitably identifying the target analyte), high sensitivity (determining low levels or the lack of the analyte), fast results (from minutes to hours), extended shelf life and easy-to-operate capabilities. However, when it comes to cost-efficient devices these features are often conflicting. Interestingly, nanomaterials enable us to engineer and manipulate unprecedented biosensing systems based on transducing phenomena occurring at the nanoscale. Thus, the outstanding features and new possibilities offered by nanomaterials are opening the way to 


\section{WILEY-VCH}

exciting opportunities to overcome this challenge. In addition, we believe that flexible, abundant and low-cost substrates, that is, plastic and paper can boost such opportunities and their simplicity.

Given their advantageous properties and functionalities, graphene derivatives are twodimensional nanoscale materials that are under active research and the research community has devoted great effort and many resources to the development of graphene-based biosensors. In fact, a myriad of analytes including bacteria, viruses, proteins, oligonucleotides, small molecules and metal ions have been successfully detected using graphene-based biosensors at the cutting edge. ${ }^{[1,2]}$ Due to the excellent properties of graphene derivatives -such as excellent capabilities for direct wiring with biomolecules, a heterogeneous chemical and electronic structure, the possibility to be processed in aqueous suspension and the ability to be tuned as insulator, semiconductor or semi-metal; graphene-based biosensors possess a plethora of advantages over other nanomaterials-based sensors, facilitating unique biosensing approaches, which will be discussed throughout this essay.

One should not be misled into thinking that graphene is a single material enjoying always the same properties and performance. On the contrary, graphene has a wide family of materials that offers a myriad of behaviors. In general, graphene derivatives possess high specific surface area $\left(>470 \mathrm{~m}^{2} \mathrm{~g}^{-1}\right)$ and broad chemical-modification capabilities. However, it should be remarked that their specific mechanical, electrical and optical properties depend on different parameters such as their purity degree, structural defects, lateral size, number of layers and oxidation level, which are strongly related to their production method. Hence, graphene derivatives exploited in biosensing are expected to be judiciously selected according to the targeted application and different questions may raise; for instance, what are the most common graphene derivatives in electrical / optical biosensing?, how do the aforementioned 


\section{WILEY-VCH}

parameters affect biosensing behavior?. We will critically address these questions and expose our perspective on the integration of graphene derivatives into plastic and paper based substrates (Figure 1).

\section{Graphene derivatives and their properties facilitating biosensing platforms}

Pristine graphene, polycrystalline graphene, graphene quantum dots (GQDs) and (reduced) graphene oxide are the most common graphene derivatives used in biosensing. Pristine graphene is a flawless lattice of $\mathrm{sp}^{2}$-bonded carbon atoms, that is, without defects throughout its honeycomb-like pattern, and it is a single crystalline grain. ${ }^{[3]}$ Polycrystalline graphene is composed of single-crystalline graphene grains of distinct orientations with a mosaic-like organization denoted by grain boundaries, which leads to topological defects such as dislocations. ${ }^{[4]}$ GQDs are nanocrystals of graphene that are tiny sufficient to trigger exciton confinement and quantum size effect (generally GQDs show diameters of less than $20 \mathrm{~nm}$ ). As a result, GQDs have photoluminescent properties which can be tuned according to their size and surface chemistry. Graphene oxide (GO) is a lattice of $\mathrm{sp}^{2}$-bonded carbon atoms incorporating defects since it is disrupted by $\mathrm{sp}^{3}$ carbon bonds and possesses oxygencontaining groups - carboxyl groups exposed on the edges and hydroxyl and epoxy groups on the basal plane. ${ }^{[5]}$ Hence, GO often has some hydrophilic islands onto its basal plane according to its oxidation degree. ${ }^{[6]}$ Reduced graphene oxide (rGO) is composed of GO after being reduced through either a chemical or physical method. Depending on the predominance of the aforementioned hydrophilic islands, $\mathrm{GO}$ and $\mathrm{rGO}$ can be endowed with water dispersibility properties. Therefore, GO and rGO micro/nano sheets can be manipulated and applied in a water-based suspension form, which is often crucial in biosensing. Importantly, binding interactions between graphene derivatives and biomolecules such as single stranded DNA are influenced by the $\mathrm{C} / \mathrm{O}$ ratio of the employed graphene derivative -high $\mathrm{C} / \mathrm{O}$ ratios bind more strongly to biomolecules than those low $\mathrm{C} / \mathrm{O}$ ratios. ${ }^{[7]}$ Moreover, the lateral size of graphene derivatives modulates kinetics and capacity of biomolecules adsorption. ${ }^{[8]}$

Graphene derivatives incorporate inherent features such as purity degree, defects and functional groups according to their synthesis route, which are directly related to their performance and production cost. For instance, pristine graphene has remarkable properties based on its perfect crystalline structure. On the other hand, its production is hardly scalable 


\section{WILEY-VCH}

and with high production cost. Hence, a balance between production cost and performance is of major importance for industrial applications and to implement graphene based devices in day-life technologies. This serious concern has been deeply discussed in the literature. ${ }^{[9-11]}$

\subsection{Electrical-based platforms}

Heterogeneous electron transfer (HET) in graphene derivatives is correlated with the oxygen content, the amount of defects, the edge like architecture, the thickness and the impurities. Unlike pristine graphene or polycrystalline graphene, GO is highly disordered and contains different functional groups according to its production method. rGO, reduced either chemically, thermal o electrochemically, can still have oxygen-containing functional groups and can suffer from a higher level of disorder or defect areas due to the reduction process. The increase of defects in the structure increases HET, whereas the presence of oxygen functional groups decreases HET. Hence, effective reduction approaches are an important feature in graphene-based thin films. Laser scribed reduction for instance is highly effective and in addition increase the edge like structures available within the production of a foam like structure with high surface area. ${ }^{[12]}$ The reduction process not only can induce different structures, which can increase surface area, but also printing methods. Vacuum filtrated-based thin films are characterized by their well oriented GO sheets thin films whereas drop-casting or ink-jet printing induces higher edge-like structures by drop spontaneous drying process. On the other hand, in vacuum filtration the printing of GO-based films over rough substrates tend to change the film structure; and printing rGO using the same technique results in an increase in edge like structure possibly due to the rGO suspended sheets in solution that tend to aggregate or have different mechanical and hydrophobic properties in comparison with

GO. ${ }^{[13,14]}$ In the literature, it is hard to define different graphene-based structures in electrical biosensing platforms due to the lack of surface characterization; however, increased surface areas and defects have been widely described in a myriad of graphene-derived sensors to perform better than planar or defect free structures such as pristine graphene. ${ }^{[15,16]}$

Electrochemical sensors can be read-out either using voltammetric, impedimetric or potentiometric techniques. In voltammetric measurements, the electrode potential is controlled to drive an electron transfer reaction with a measured current associated to this. This can be done taking advantage for example of $\left[\mathrm{Fe}(\mathrm{CN})_{6}\right]^{3-}$ or metals where the shift is associated to a quantitative perturbation. Impedimetric measurements monitors changes in the 


\section{WILEY-VCH}

interfacial properties of the electrode surface and do not require labels generating signals to detect biorecognition events. ${ }^{[2]}$ Potentiometric technique also is an important tool in graphene biosensors due to the high-mobility of either electrons and holes in graphene. The semimetallic behavior of graphene enables to tune charge carriers from electrons to holes using external electric fields enabling to determine the Dirac point and the doping type of graphene field effect transistors (FET). This is very important in biosensors for DNA analysis or protein detection where the attachment of DNA or proteins can lead to a shift in the Dirac point enabling quantitative detection of loaded molecules. This is attributed to a change in the surface charge that can be related to the presence of a protein at a certain $\mathrm{pH}$ or to the length of the targeted DNA, inducing a higher or lower charge density resulting from the doping of these charged compounds to graphene. This methodology have been used for $\mathrm{pH}$ sensors, cell based sensors or cancer biomarkers sensing platforms. Solution-gated FET reported use of pristine or polycrystalline graphene, and recently RGO have been tested reporting characteristics similar to polycrystalline or pristine graphene. ${ }^{[17-19]}$ This could be a stepforward in the development of low cost graphene based devices.

A plethora of scientific literature on graphene electrochemistry relies on GO or rGO basedsuspensions which are drop-casted or electrodeposited onto underlying electrodes, where basically the oxidized graphene derivatives are acting in synergy with the underlying electrodes, producing summative electrochemical effects. This has also been achieved for a myriad of electrochemical (bio)sensors that are graphene-modified using screen printed carbon electrodes (SPCE), gold or indium tin oxide (ITO). In plastic-based biosensors, our research group has also performed this kind of sensors reaching advantageous tunable electrochemical properties in a SPCE modified with GO and rGO for enzyme (ex. tyrosinase) immobilization and drug detection, respectively, (Figure 2A-B) ${ }^{[20,21]}$ Similarly, in paperbased electrochemical biosensors, $\mathrm{rGO}$ has been used for tuning the electrochemical properties of microfluidic paper-based analytical devices for the detection of four different cancer biomarkers. ${ }^{[22]}$ This was achieved using photoresist-patterned paper followed by screen printing of carbon and $\mathrm{Ag} / \mathrm{AgCl}$ and additionally the drop casting of graphene and nanoparticles. Despite the low cost described by the authors, the fabrication process can be seen as a time and labor-consuming method.

Electrical biosensors exploiting rGO as a transducer are also able to exhibit advantageous performance either in plastic or paper architectures. In fact, rGO-based flexible field effect 


\section{WILEY-VCH}

transistors printed on plastic exhibit a performance comparable with those based on pristine graphene which represents a good alternative for scalable bio applications of rGO thin films transistors. ${ }^{[18]}$ In general, electrical biosensors based on the combination of GO or rGO and plastic are making an evolution in performance and production cost. However, polycrystalline graphene-based biosensors are making one step forward for portable and wearable biosensors that until now cannot be observed in GO or rGO electrochemical bio-devices with implemented human-machine interfaces and enabling theranostics. ${ }^{[23,24]}$

In the paper based field, ink jet printing is playing an important role for the device production which could be related with the opportunity to pattern GO or rGO-based inks without changing the features of the paper in a simple fashion and enabling the improvement of the conductivity via UV-pulsed laser irradiation. ${ }^{[25]}$ Other deposition techniques implying pressure are prone to change the paper thickness and its fluidics. It is worth mentioning that and in spite of the large surface roughness of conventional printing paper, graphene-based (opto)electronic devices can also be fabricated using this substrate, which could be of interest for innovative biosensing. In fact, multilayer graphene (growth via chemical vapor deposition) has been demonstrated to be transferred to conventional paper taking advantage of the hydrophobic character of multilayer polycrystalline graphene. ${ }^{[26]}$

The production of graphene-based thin films is of major importance to a wide variety of applications such as electrical and electrochemical sensors. Their low cost and simplicity could improve the fast integration and abundance of graphene-based devices. Recently our research team reported a simple way to transfer GO patterned structures to target substrates by simple water activation and pressure using wax printed membranes, (Figure $2 \mathrm{C}$ ). ${ }^{[13]}$ Since the wax printing technology is a cheap and versatile technique, one can produce unlimited shapes and structures and the control over the thickness provided by the vacuum filtration enables to print nanometer vertically sized films. This represents an interesting alternative to existing patterning technologies such as screen printing, gravure, inkjet, microcontact stamps and lithography. In fact, we are able to print over paper, textile or plastics opening venues for lowcost and flexible all-graphene based biosensors overall in cases where the size of the sample to be analyzed is not a major issue.

\subsection{Optical-based platforms}




\section{WILEY-VCH}

Graphene derivatives are optically omnivorous, that is, their fluorescence quenching capabilities are virtually universal and they are able to quench any fluorescence wavelength via energy transfer. It is noteworthy that not only their number of layers determines the efficiency of their fluorescence quenching ability, ${ }^{[27]}$ but also their oxidation degree affects their quenching factor - the more the utilized GO is oxidized, the less efficient it becomes in terms of fluorescence quenching capabilities. ${ }^{[7]}$ In addition, long range energy transfer is also known to occur in graphene derivatives, which is observable up to $30 \mathrm{~nm}{ }^{[28]}$

Oxidized graphene derivatives undergo a recombination of electron-hole pairs localized within a $\mathrm{sp}^{2}$ carbon domain embedded within a $\mathrm{sp}^{3}$ matrix, resulting in photoluminescent properties. Their maximum emission wavelength can be modulated from the blue region to the near-infrared region in accordance with their oxygen-containing moieties, ${ }^{[29]}$ lateral size ${ }^{[30]}$ and oxidation degree. ${ }^{[31]}$ Although the synthesis of photoluminescent graphene derivatives endowed with high quantum yield has been scarcely developed (e.g. >70\%), nitrogen doping has been reported to be an effective route to address this paucity and improve such an important parameter. $^{[32]}$

The most common graphene-based optical biosensing principle relies on the interaction between single stranded DNA (ssDNA) and graphene derivatives, which is facilitated by hydrophobic interactions and the strong $\pi-\pi$ stacking interaction between the hexagonal cells of graphene derivatives and the ring structures in nucleotides. This leads to a simple, yet sensitive and versatile optical biosensing mechanism: in the absence of the analyte a fluorescent dye-labeled ssDNA is complexed and quenched via the interaction with a graphene derivative, whereas the complex dye-ssDNA/graphene is detached in the presence of the analyte. Consequently, the dye-labeled ssDNA recovers its fluorescence as graphene derivatives weakly interact with the rigid structure of double stranded DNA or aptamer-target complexes. GQDs, and r/GO micro and nanosheets are the most common graphene derivatives in optical biosensing, whereas graphene-based optical biosensing is mainly capitalizing on the aforementioned photonic phenomenon.

Our research group has explored the photoluminescence quenching capabilities and the oxygenated two-dimensional network of GO in order to exploit it as a pathogen-revealing agent. ${ }^{[28]}$ To this aim, we used a conventional glass slide-based microarray system, whose limit of detection is 760 times more sensitive than a conventional immunoassay, though 


\section{WILEY-VCH}

expensive, relatively time-consuming and not particularly amenable to portability (Figure 3A). We then demonstrated an innovative technological platform that transfers the previous microarray-based platform into a paper-based lateral flow immunoassay for bacteria detection -in particular Escherichia coli, as a model pathogen. This biosensing approach eliminates the use of secondary antibodies and is revealed by the photoluminescence quenching capability of GO. The sensing principle is based on the energy transfer phenomenon that occurs between photoexcited quantum dots (QDs) and GO while being in close proximity. The analyte is selectively attached onto the test line of the strip (which contains antibody-decorated QDs) leading to a highly specific and sensitive sensing phenomenon that is clearly observable up to $10 \mathrm{CFU} \mathrm{mL} \mathrm{m}^{-1}$ in standard buffer and $100 \mathrm{CFU} \mathrm{mL}^{-1}$ in bottled water and milk. The control line of the strip containing bare QDs is always quenched upon GO addition, indicating the correct operation of the system (Figure 3B, upper part). As a cost-effective and easy-to-use approach, this device is appealing for portable and automated diagnostics applications that may include other similar size analytes with interest for diagnostics. ${ }^{[33]}$ Furthermore, we successfully transferred this nanoenabled approach to another flexible, lightweight an optically transparent substrate such as bacterial cellulose nanopaper (Figure 3B, lower part). ${ }^{[34]}$

We have also exploited GQDs to engineer an innovative composite material targeting the detection of small molecules such as a pesticide [tributyltin (291 Da)]. The hybrid material consists of magnetic silica beads encapsulated by GQDs embedded within molecularly imprinted polypyrrole. Thus, the mSGP composite is endowed with: i) magnetic properties, which are useful for separation and pre-concentration of the toxic compound; ii) water dispersibility, which is important to perform a liquid-phase-based assay; iii) selectivity, which is crucial to detect exclusively the target analyte and iv) photoluminescence as an optical transducing system that is quenched via energy transfer upon analyte binding. Gathering these features, such a GQDs-based sensing platform enables a rapid, simple and sensitive approach for small molecule detection even in complex media, e.g. marine water, without any sample treatment. ${ }^{[35]}$

\section{Simple biosensors based on the integration of graphene derivatives into low-cost, lightweight and flexible substrates: Discussion and Future Outlooks}

Graphene derivatives compete with other well-known materials (e.g. silicon, germanium and ITO). However, this novel family of materials is likely to find its best opportunities in 


\section{WILEY-VCH}

innovative areas, that is, functions, applications, products or technologies not dominated by other conventional materials. ${ }^{[36]}$ Aside from this, currently, graphene market is mainly focused on graphene derivatives production, whereas some opto/electronic devices such as field effect transistors, magnetic sensors, and photodetectors have also been launched. By contrast, development of final products involving graphene-based biosensors is an emergent area. Thus, in the mid-term, graphene-based biosensors are likely to find excellent opportunities and a great market niche within this context.

Well-established technologies allowing for diagnostics, drug discovery, food safety, defense, security, and environmental monitoring often require bulky infrastructure, conventional electrical supply, time-consuming and labor-intensive procedures and qualified personnel. Aiming at simplifying such technologies and manufacturing easy-to-use, yet powerful and innovative biosensing devices, we envision a new generation of biosensing devices based on the synergy between flexible, lightweight, easy-to-use, versatile and cost-effective materials (paper and plastic) and the outstanding properties of graphene derivatives.

When graphene derivatives are combined with cheap, lightweight and flexible substrates, interesting opportunities arise. Flexibility facilitates advantages in terms of size and weight of the engineered biosensor. Moreover, flexibility obviates bulky architectures, facilitates assembling processes and allows for the generation of wearable and implantable devices. Aside from their flexibility, abundance and low-cost; plastic and paper can be readily modified to create breakthrough devices using suspensions of graphene derivatives and a myriad of deposition techniques, including drop-casting, dip-coating, spraying, spin-coating, inkjet printing, screen printing, high-volume roll-to-roll gravure printing, pressure based mechanisms, etc. Among these innovative biosensors created through the combination of graphene derivatives and flexible substrates we could find advantageous theranostic platforms, point of care devices, wearable devices and omnipresent biosensors targeting different tasks involved with diverse applications like healthcare, therapy, diagnostics, agriculture, environment, food safety and security. The tuning with new electronic and photonic functions in flexible substrates might be a future in new technologies based on graphene derivatives for autonomous biosensing devices with integrated photonics, electronics and microfluidics. On the other hand, we believe there is still much to discover and develop within the field of this kind of biosensors. For instance, GO or rGO membranes, also termed as GO or rGO papers, are making a step forward in the supercapacitor field. ${ }^{[37]}$ This membranes have also been 


\section{WILEY-VCH}

tested for molecular sieving which could be applied in the generation of breakthrough biosensing architectures. ${ }^{[38,39]}$ Although analytical lab-on-a-chip applications capitalizing on graphene derivatives properties are not widely reported in the literature, one can find encouraging approaches facilitating electrochemical amplification mechanisms, multiplexed optical detection strategies and a novel crystallographic analysis, ${ }^{[40-42]}$ which are an excellent example of how the thickness, conductivity and transparency offered by graphene derivatives represent a powerful tool for lab-on-a-chip development"

Solving real-world problems through simple — albeit powerful, safe and robust — biosensors based on the integration of graphene derivatives into cheap and flexible substrates will enforce the successful transition of these innovative devices to the market. Moreover, the novel insulating, semiconducting and optoelectronic properties provided by other exciting 2D materials (hexagonal boron nitride, metal dichalcogenides, black phosphorus) and their combination is also expected to bring breakthrough approaches solving real-world problems through biosensing. However, overall commercialization of graphene-based biosensors will imply overcoming diverse technical, production and market issues. Here we spot some of these crucial issues:

- Relevant stakeholders in the biosensing field demand different challenging characteristics, including but not limited to miniaturization potential, low powerbudget $(\sim \mu \mathrm{W}-\mathrm{mW})$, robust shelf and operational lifetime stability, re-calibration capabilities (as they may suffer from temperature or relative humidity drifts) and mechanical shock resistance.

- The performance reproducibility of graphene-based biosensors strongly depend on homogeneity of the quality of the involved graphene derivatives, whose manufacturing scalability is not particularly easy to control, above all when it comes to devices relying on a single flake such as bio-field effect transistors. Moreover, sensitivity and specificity (overall in clinical diagnostics), which are seldom reported in the literature related to biosensors, should be systematically determined in terms of percentage of false negatives and positives, respectively. ${ }^{[43]}$

- Graphene deposition techniques yield different coating qualities in terms of surface coverage and interconnection between graphene flakes, which should be judiciously 


\section{WILEY-VCH}

considered along with substrate roughness and morphology of the individual flakes; especially in electrical approaches, where conductivity is heavily affected by these features. Firstly, single layers undergo a folding and wrinkling effect to prevent collapsing into multilayers. ${ }^{[38,39]}$ Secondly, some graphene deposition techniques tend to promote an extreme degree of such a folding and wrinkling effect (e.g. drop casting and spin coating, which reduce surface coverage and increase the roughness, deteriorating electrical performance). By contrast, other graphene deposition techniques are amenable to performing a large-area monolayer of flakes with much higher surface coverage, leading to conducting thin films. ${ }^{[44]}$

- Although thoroughly systematic and well-designed experiments reveal encouraging guidance for the use of graphene in biological environments, ${ }^{[45,46]}$ graphene derivatives toxicity is still under debate overall in the case of wearable platforms.

- Corresponding/right validation and evaluation in real settings and ongoing proficiency-testing of graphene-based biosensors are crucial to demonstrate advantages over well-known sensing technologies in terms of simplicity, sensitivity, specificity, operating time or cost.

All in all, graphene-based biosensing performance heavily depends on purity degree, lateral size, number of layers, oxidation level, distribution of oxygen-containing moieties, and/or defects of the employed graphene derivatives since these features often modulate electrical conductivity, fluorescence quenching capabilities, photoluminescence, binding interactions (between graphene derivatives and biomolecules) and kinetics and capacity of biomolecules adsorption. Hence, scientists and technologists working with graphene-based biosensors are strongly encouraged to consider and characterize all these features judiciously with the aim to standardize the quality of graphene derivatives in biosensing and ensure reproducibility of this novel type of biosensors.

Getting their best opportunities in applications not dominated by conventional materials, simple graphene-based biosensors will lead to breakthrough solutions for the real world.

\section{Acknowledgements}




\section{WILEY-VCH}

The authors acknowledge the support from H2020-EU (INTCATCH Project, Ref. 689341), MINECO (Spain, MAT2014-52485-P, RTC-2014-2619-7 and Severo Ochoa Program, Grant SEV-2013-0295) and Secretaria d' Universitats i Recerca del Departament d' Economia i Coneixement de la Generalitat de Catalunya (2014 SGR 260)

Received: ((will be filled in by the editorial staff))

Revised: ((will be filled in by the editorial staff)) Published online: ((will be filled in by the editorial staff))

References

[1] E. Morales-Narváez, A. Merkoçi, Adv. Mater. 2012, 24, 3298.

[2] A. Ambrosi, C. K. Chua, N. M. Latiff, A. H. Loo, C. H. A. Wong, A. Y. S. Eng, A. Bonanni, M. Pumera, Chem. Soc. Rev. 2016, 45, 2458.

[3] L. Rodríguez-Pérez, M. Á. Herranz, N. Martín, Chem. Commun. 2013, 49, 3721.

[4] O. V Yazyev, Y. P. Chen, Nat. Nanotechnol. 2014, 9, 755.

[5] D. R. Dreyer, S. Park, C. W. Bielawski, R. S. Ruoff, Chem. Soc. Rev. 2010, 39, 228.

[6] S. Eigler, A. Hirsch, Angew. Chem. Int. Ed. Engl. 2014, 53, 7720.

[7] B. J. Hong, Z. An, O. C. Compton, S. T. Nguyen, Small 2012, 8, 2469.

[8] J. Lee, Y. Yim, S. Kim, M.-H. Choi, B.-S. Choi, Y. Lee, D.-H. Min, Carbon N. Y. 2016, 97, 92.

[9] K. S. Novoselov, V. I. Fal[prime]ko, L. Colombo, P. R. Gellert, M. G. Schwab, K. Kim, Nature 2012, 490, 192.

[10] W. Ren, H.-M. Cheng, Nat Nano 2014, 9, 726.

[11] A. Zurutuza, C. Marinelli, Nat Nano 2014, 9, 730.

[12] M. F. El-Kady, R. B. Kaner, Nat Commun 2013, 4, 1475.

[13] L. Baptista-Pires, C. C. Mayorga-Martínez, M. Medina-Sánchez, H. Montón, A. Merkoçi, ACS Nano 2016, 10, 853.

[14] W. J. Hyun, O. O. Park, B. D. Chin, Adv. Mater. 2013, 25, 4729.

[15] K. Griffiths, C. Dale, J. Hedley, M. D. Kowal, R. B. Kaner, N. Keegan, Nanoscale 2014, 6, 13613. 


\section{WILEY-VCH}

[16] A. Salehi-Khojin, D. Estrada, K. Y. Lin, M.-H. Bae, F. Xiong, E. Pop, R. I. Masel, Adv. Mater. 2012, 24, 53.

[17] R. X. He, P. Lin, Z. K. Liu, H. W. Zhu, X. Z. Zhao, H. L. W. Chan, F. Yan, Nano Lett. 2012, 12, 1404.

[18] Q. He, H. G. Sudibya, Z. Yin, S. Wu, H. Li, F. Boey, W. Huang, P. Chen, H. Zhang, ACS Nano 2010, 4, 3201.

[19] S. Liu, X. Guo, NPG Asia Mater 2012, 4, e23.

[20] L. Baptista-Pires, B. Pérez-López, C. C. Mayorga-Martinez, E. Morales-Narváez, N. Domingo, M. J. Esplandiu, F. Alzina, C. M. S.- Torres, A. Merkoçi, Biosens. Bioelectron. 2014, 61, 655.

[21] S. Kurbanoglu, L. Rivas, S. A. Ozkan, A. Merkoçi, Biosens. Bioelectron. n.d., DOI http://dx.doi.org/10.1016/j.bios.2016.07.109.

[22] Y. Wu, P. Xue, Y. Kang, K. M. Hui, Anal. Chem. 2013, 85, 8661.

[23] H. Lee, T. K. Choi, Y. B. Lee, H. R. Cho, R. Ghaffari, L. Wang, H. J. Choi, T. D. Chung, N. Lu, T. Hyeon, S. H. Choi, D.-H. Kim, Nat Nano 2016, 11, 566.

[24] M. S. Mannoor, H. Tao, J. D. Clayton, A. Sengupta, D. L. Kaplan, R. R. Naik, N. Verma, F. G. Omenetto, M. C. McAlpine, Nat Commun 2012, 3, 763.

[25] S. R. Das, Q. Nian, A. A. Cargill, J. A. Hondred, S. Ding, M. Saei, G. J. Cheng, J. C. Claussen, Nanoscale 2016, 8, 15870.

[26] E. O. Polat, H. B. Uzlu, O. Balci, N. Kakenov, E. Kovalska, C. Kocabas, ACS Photonics 2016, 3, 964.

[27] Z. Chen, S. Berciaud, C. Nuckolls, T. F. Heinz, L. E. Brus, ACS Nano 2010, 4, 2964.

[28] E. Morales-Narváez, A.-R. Hassan, A. Merkoçi, Angew. Chemie Int. Ed. 2013, 52, 13779.

[29] R. M. and A. M. and C. N. and S. K. Ray, Nanotechnology 2014, 25, 495704.

[30] M.-H. Jang, H. D. Ha, E.-S. Lee, F. Liu, Y.-H. Kim, T. S. Seo, Y.-H. Cho, Small 2015, 


\section{WILEY-VCH}

$11,3773$.

[31] Q. Mei, J. Chen, J. Zhao, L. Yang, B. Liu, R. Liu, Z. Zhang, ACS Appl. Mater. Interfaces 2016, 8, 7390 .

[32] J. Sun, S. Yang, Z. Wang, H. Shen, T. Xu, L. Sun, H. Li, W. Chen, X. Jiang, G. Ding, Z. Kang, X. Xie, M. Jiang, Part. Part. Syst. Charact. 2015, 32, 434.

[33] E. Morales-Narváez, T. Naghdi, E. Zor, A. Merkoçi, Anal. Chem. 2015, 87, 8573.

[34] E. Morales-Narváez, H. Golmohammadi, T. Naghdi, H. Yousefi, U. Kostiv, D. Horák, N. Pourreza, A. Merkoçi, ACS Nano 2015, 9, 7296.

[35] E. Zor, E. Morales-Narváez, A. Zamora-Gálvez, H. Bingol, M. Ersoz, A. Merkoçi, ACS Appl. Mater. Interfaces 2015, 7, 20272.

[36] M. Peplow, Nature 2013, 503, 327.

[37] R. Raccichini, A. Varzi, S. Passerini, B. Scrosati, Nat Mater 2015, 14, 271.

[38] B. Mi, Science (80-. ). 2014, 343, 740 LP.

[39] J. Gaughran, D. Boyle, J. Murphy, R. Kelly, J. Ducrée, Microsystems Nanoeng. 2016, 2, 16008.

[40] Y. Ueno, K. Furukawa, K. Matsuo, S. Inoue, K. Hayashi, H. Hibino, Anal. Chim. Acta 2015, $866,1$.

[41] L. Syedmoradi, M. Daneshpour, M. Alvandipour, F. A. Gomez, H. Hajghassem, K. Omidfar, Biosens. Bioelectron. 2017, 87, 373.

[42] S. Sui, Y. Wang, K. W. Kolewe, V. Srajer, R. Henning, J. D. Schiffman, C.

Dimitrakopoulos, S. L. Perry, Lab Chip 2016, 16, 3082.

[43] W. C. W. Chan, B. Udugama, P. Kadhiresan, J. Kim, S. Mubareka, P. S. Weiss, W. J. Parak, ACS Nano 2016, 10, 8139.

[44] L. J. Cote, F. Kim, J. Huang, J. Am. Chem. Soc. 2009, 131, 1043.

[45] K. Kostarelos, K. S. Novoselov, Science 2014, 344, 261.

[46] D. A. Jasim, C. Ménard-Moyon, D. B?gin, A. Bianco, K. Kostarelos, Chem. Sci. 2015, 


\section{WILEY-VCH}

$6,3952$.

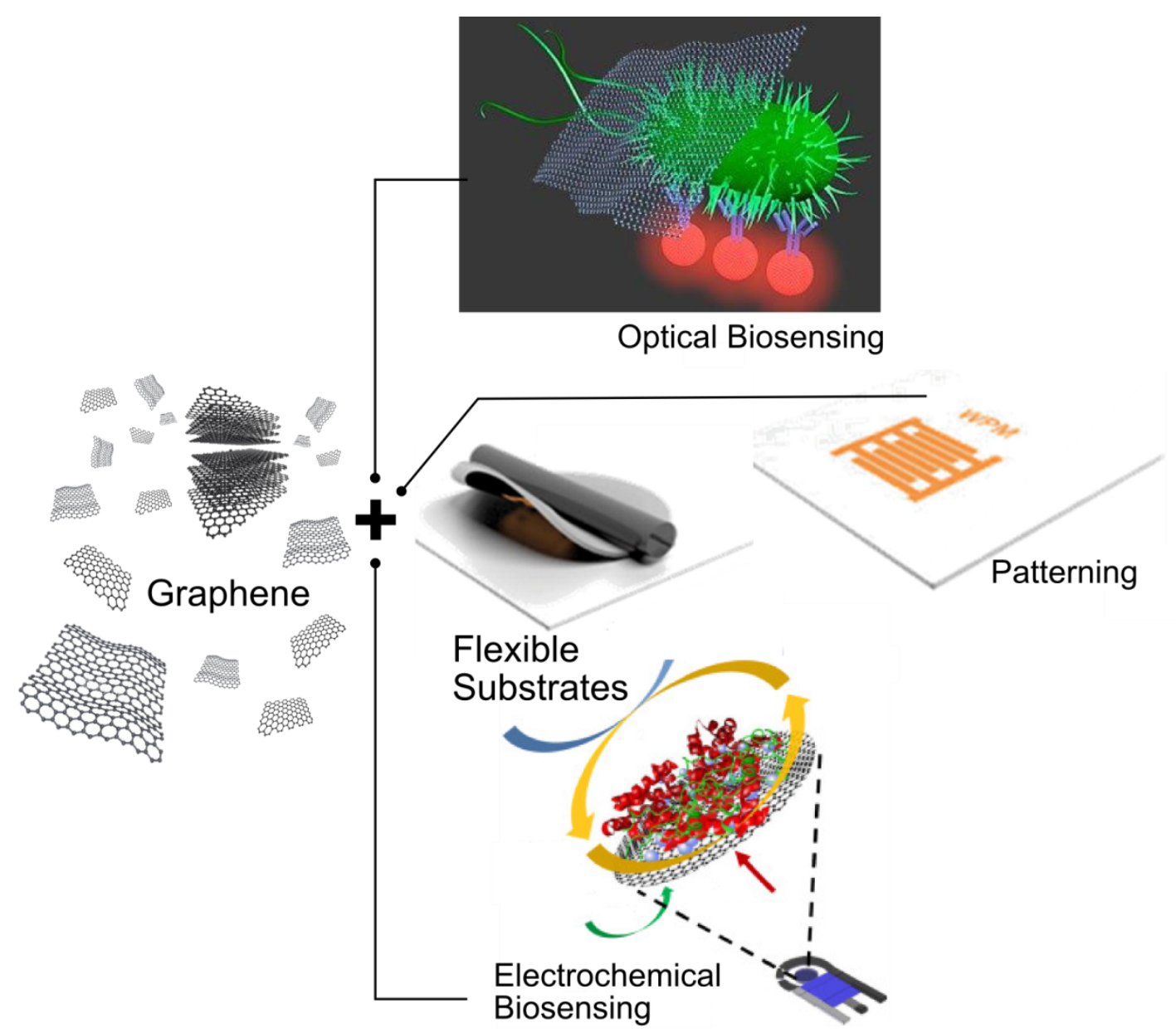

Figure 1. Integration of graphene derivatives into plastic and paper-based substrates as a biosensing platform. We envision a new generation of biosensing devices based on the synergy between flexible, lightweight, easy-to-use, versatile and cost-effective materials (paper and plastic) and the outstanding properties of graphene derivatives.
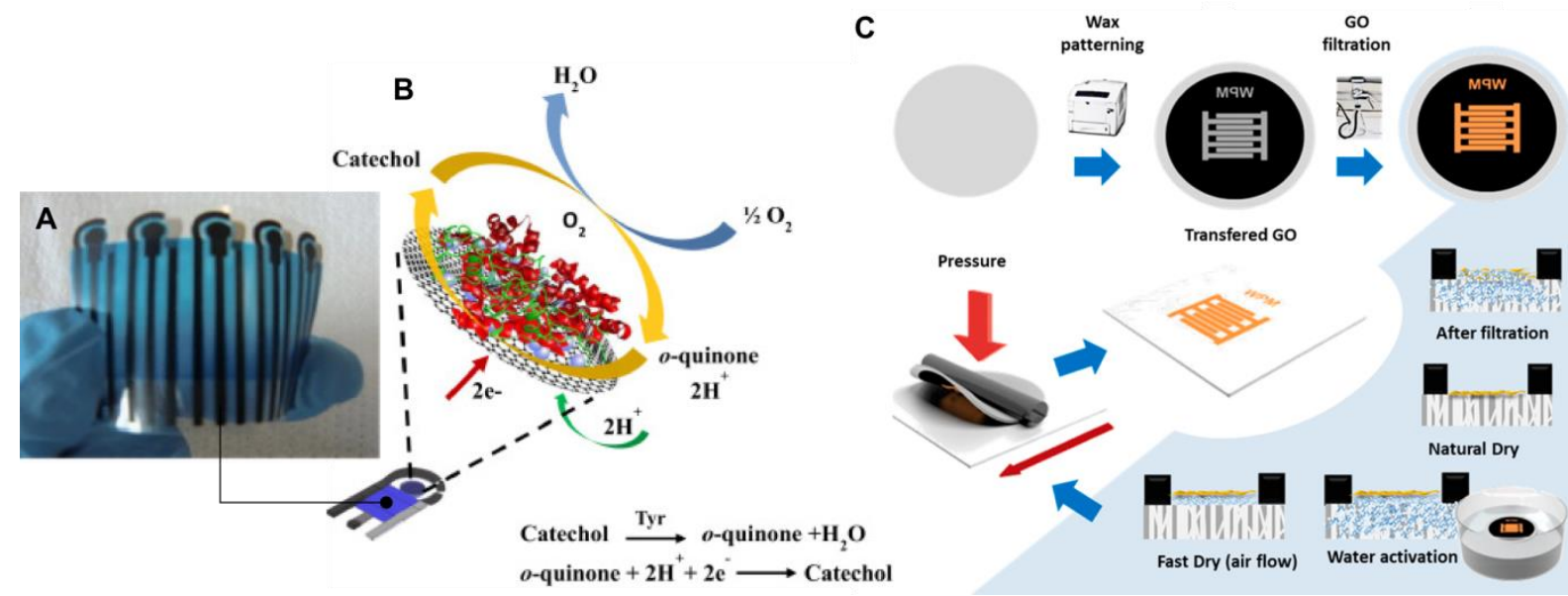

Figure 2. A. Electrodes printed on plastic (PET) via screen printing method and (B) modified with graphene. A. Adapted with permission. ${ }^{[20]}$. Copyright $\bigodot(2014)$ Elsevier. Adapted with 


\section{WILEY-VCH}

permission. ${ }^{[21]}$ Copyright (C) (2016) Elsevier. C. Simple patterning of graphene. Adapted with permission. ${ }^{[13]}$. Copyright (C) (2016) American Chemical Society, respectively.

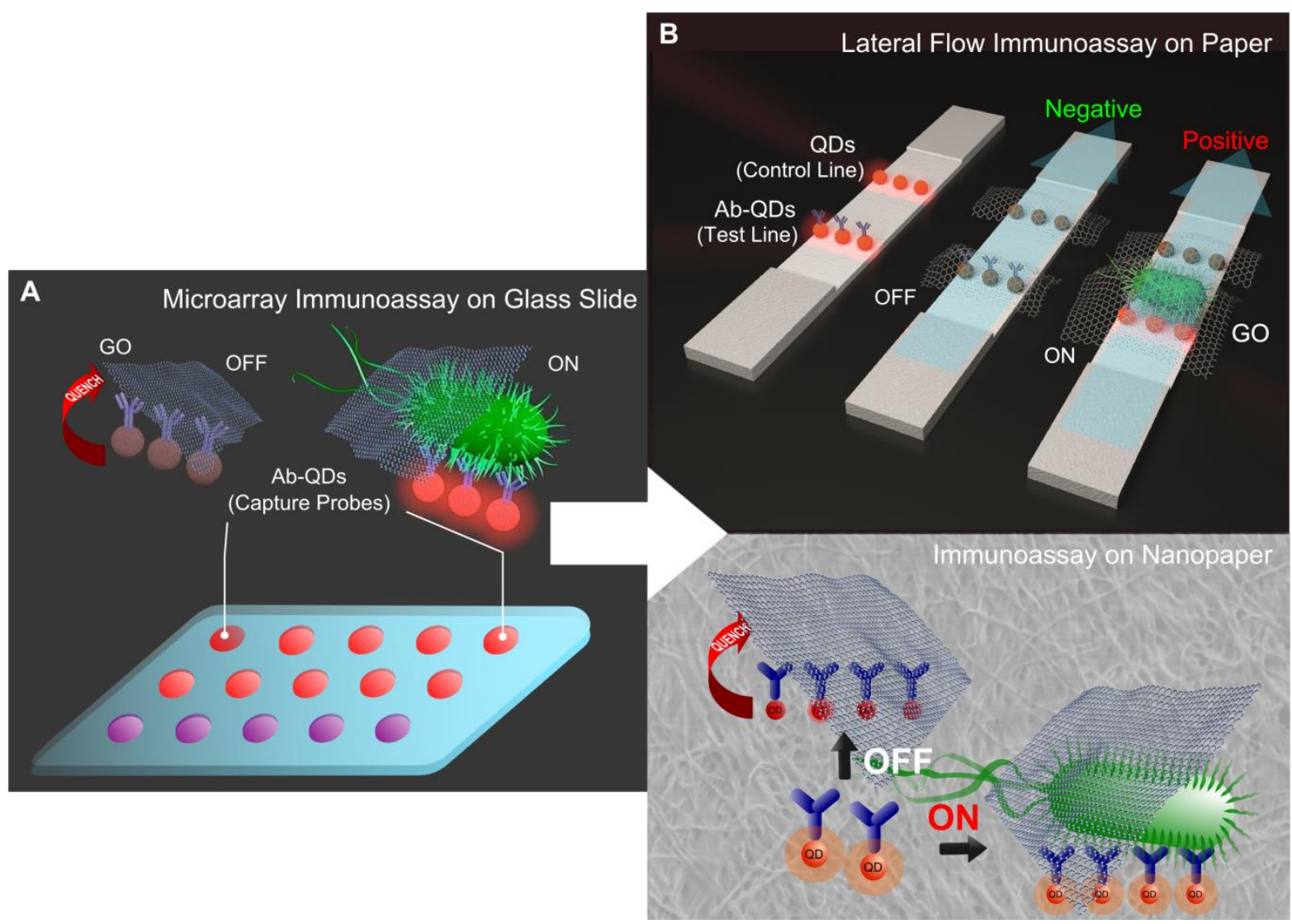

Figure 3. Graphene oxide as a pathogen-revealing agent. A. Microarray immunoassay revealed by GO on glass slide. Adapted with permission from ref. ${ }^{[28]}$. Copyright $@(2013)$ WILEY-VCH Verlag GmbH \& Co. KGaA, Weinheim. B. Upper part, lateral flow immunoassay revealed by GO on paper. Adapted with permission from ref. ${ }^{[33]}$ Copyright (C) (2015) American Chemical Society. B. Lower part, immunoassay revealed by GO on bacterial cellulose nanopaper. Adapted with permission from ref. ${ }^{[34]}$ Copyright $\odot ~(2015)$ American Chemical Society. 


\section{WILEY-VCH}

We discuss our perspective on a new generation of biosensing devices based on the synergy between flexible, lightweight, easy-to-use, versatile and cost-effective materials (paper and plastic) and the outstanding properties of graphene derivatives

\section{Biosensing}

E. Morales-Narváez, L. Baptista-Pires, A. Zamora-Gálvez, A. Merkoçi*

Graphene-based biosensors: Going simple

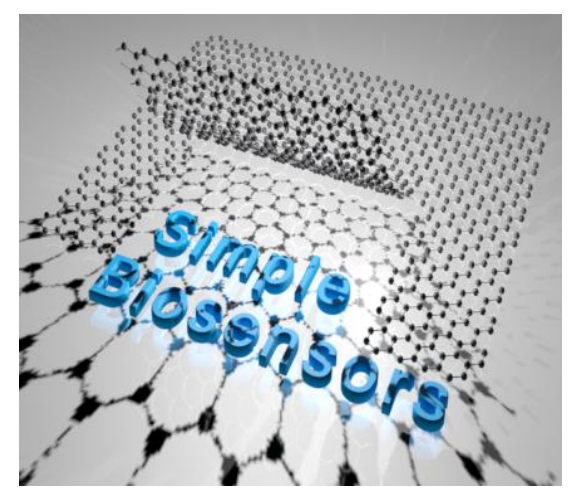

